\title{
Non-Markovian quantum state diffusion for an open quantum system in fermionic environments
}

\author{
Mi Chen ${ }^{1}$ and J. Q. You ${ }^{2}$ \\ ${ }^{1}$ Department of Physics and State Key Laboratory of Surface Physics, Fudan University, Shanghai 200433, China \\ ${ }^{2}$ Beijing Computational Science Research Center, Beijing 100084, China
}

(Dated: August 25, 2018)

\begin{abstract}
Non-Markovian quantum state diffusion (NMQSD) provides a powerful approach to the dynamics of an open quantum system in bosonic environments. Here we develop an NMQSD method to study the open quantum system in fermionic environments. This problem involves anticommutative noise functions (i.e., Grassmann variables) that are intrinsically different from the noise functions of bosonic baths. We obtain the NMQSD equation for quantum states of the system and the non-Markovian master equation. Moreover, we apply this NMQSD method to single and double quantum-dot systems.
\end{abstract}

\section{INTRODUCTION}

The theory of open quantum systems has become an increasingly important topic in, e.g., quantum information science, quantum measurement, and quantum optics. Traditionally, the dynamics of an open quantum system was often investigated using a Markov master equation derived by invoking the Born-Markov appoximation. However, this formalism fails for many solid-state systems (see, e.g., [1]) where the system-environment coupling is strong and the environment is structured. Thus a non-Markovian master equation is required when considering the memory effect and back action of the environment. It is known that the derivation of an exact non-Markovian master equation has long been a challenging task. One of the breakthroughs is the exact nonMarkovian master equation for quantum Brownian motion model derived by $\mathrm{Hu}$ et al. [2] using the FeynmanVernon influence functional path-integral method [3].

Of all the theoretical strategies used to deal with open quantum systems, a non-Markovian quantum trajectory theory known as non-Markovian quantum state diffusion (NMQSD) 4, 5] provides a powerful approach to the dynamics of an open quantum system in bosonic environments. In this approach, when the so-called $O$-operator is obtained, the quantum dynamics of an open system is determined by solving the NMQSD equation (i.e., a diffusive stochastic Schrödinger equation) and the nonMarkovian master equation can also be derived [6, 7]. In contrast to the conventional master equation under the Born approximation, this non-Markovian mater equation is derived non-perturbatively, so it applies even for a strong system-environment coupling. Indeed, some exact $O$-operators have been found in a variety of quantum models [4 9], including multilevel models [10].

In addition to bosonic baths, fermionic baths are also involved in many physical systems, particularly in solidstate systems. The Feynman-Vernon influence functional path-integral method can also be used to study the quantum dynamics of an open system in a fermionic environment (see, e.g., [11]). Recently, this path-integral method was extended to derive non-Markovian master equations for nanodevices [12, 13]. Also, there were other studies on quantum dynamics and transport of nanostructured systems, including the rate-equation approach [14, 15], and the Markovian (see, e.g., [16, 17]) and non-Markovian (see, e.g., 18) master equation approaches. In addition, the nonequilibrium Green's function method can be used to study the quantum transport through the nanostructures (see, e.g., [19]). Nevertheless, the extension of NMQSD method to an open quantum system in fermionic baths has been a long-standing unsolved problem because this open system involves anticommutative noise functions (i.e., Grassmann variables) that are intrinsically different from the noise functions of bosonic baths.

In this paper, we develop an NMQSD method to study the open quantum system in fermionic baths. This NMQSD approach is formulated in a non-perturbative manner and it applies for both weak and strong systemenvironment couplings. We not only obtain the NMQSD equation for quantum states of the system, but also derive the non-Markovian master equation. Moreover, as interesting examples, we apply this NMQSD method to single and double quantum-dot systems. Note that our NMQSD method belongs to the quantum trajectory approach involving continuous time evolution. There is another kind of quantum trajectory approach which involves discontinuous time evolution, i.e., quantum jumps (see, e.g., [20]). The non-Markovian quantum trajectory approach in [21] generalizes the Markovian quantum jump method and can also apply to both bosonic and fermionic baths.

\section{QUANTUM STATE DIFFUSION EQUATION}

We consider a quantum system coupled to two fermionic baths: $H=H_{\text {sys }}+H_{\text {env }}+H_{\text {int }}$, with (we set 
$\hbar=1)$

$$
\begin{aligned}
& H_{\mathrm{env}}=\sum_{k}\left(\omega_{L k} a_{L k}^{\dagger} a_{L k}+\omega_{R k} a_{R k}^{\dagger} a_{R k}\right), \\
& H_{\mathrm{int}}=\sum_{k}\left(g_{L k} c_{L}^{\dagger} a_{L k}+g_{R k} c_{R}^{\dagger} a_{R k}+\text { H.c. }\right) .
\end{aligned}
$$

Here $H_{\text {sys }}$ denotes the Hamiltonian of the system, $H_{\text {env }}$ is the Hamiltonian of the two electric leads acting as fermionic baths, and $H_{\text {int }}$ models the interactions between the system and the two baths. The spectral density function of each bath is

$$
J_{\lambda}(\omega)=\sum_{k}\left|g_{\lambda k}\right|^{2} \delta\left(\omega-\omega_{\lambda k}\right),
$$

where $\lambda=L$ or $R$. In Eq. (1), $a_{\lambda k}^{\dagger}\left(a_{\lambda k}\right)$ is the fermionic creation (annihilation) operator for a quantum state with wave vector $k$ in the left or right lead. We assume that the system of interest couples to the two leads via single channels characterized by the fermionic creation (annihilation) operators $c_{\lambda k}^{\dagger}\left(c_{\lambda k}\right)$ [see Eq. (2)]. Extension to a multi-channel case is straightforward.

In an NMQSD approach, environments are required to be initially at zero temperature, so as to conveniently represent the environmental degrees of freedom with the coherent state basis. As for environments initially with a nonzero temperature, one can map the nonzerotemperature density operator to a zero-temperature density operator using a Bogoliubov transformation [22]. In the case of fermionic baths, this requires to add $\sum_{\lambda k} \omega_{\lambda k} b_{\lambda k} b_{\lambda k}^{\dagger}$ to Eq. (1), corresponding to the part involving holes in the electric leads. The Bogoliubov transformation for fermionic operators can be introduced as

$$
\begin{aligned}
& a_{\lambda k}=\sqrt{1-\bar{n}_{\lambda k}} d_{\lambda k}-\sqrt{\bar{n}_{\lambda k}} e_{\lambda k}^{\dagger} \\
& b_{\lambda k}=\sqrt{1-\bar{n}_{\lambda k}} e_{\lambda k}+\sqrt{\bar{n}_{\lambda k}} d_{\lambda k}^{\dagger},
\end{aligned}
$$

where $\bar{n}_{\lambda k}=\left[e^{\left(\omega_{\lambda k}-\mu_{\lambda}\right) / k_{B} T}+1\right]^{-1}$ is the average number of electrons in the $k$ th state of the left (right) electric lead with chemical potential $\mu_{\lambda}$. In Eq. (4), the coefficients are determined by the requirement that the derived master equation reduces to a Lindblad form in the Markovian limit. The transformed Hamiltonian $\mathcal{H}$ is written as

$$
\begin{aligned}
\mathcal{H}= & H_{\mathrm{sys}}+\sum_{\lambda k}\left[\omega_{\lambda k}\left(d_{\lambda k}^{\dagger} d_{\lambda k}+e_{\lambda k} e_{\lambda k}^{\dagger}\right)\right. \\
& \left.+\left(\sqrt{\bar{n}_{\lambda k}} g_{\lambda k}^{*} c_{\lambda} e_{\lambda k}+\sqrt{1-\bar{n}_{\lambda k}} g_{\lambda k} c_{\lambda}^{\dagger} d_{\lambda k}+\text { H.c. }\right)\right]
\end{aligned}
$$

where the new fermionic operator $d_{\lambda k}\left(e_{\lambda k}^{\dagger}\right)$ corresponds to the annihilation of electrons (holes) in the virtual fermionic baths. Note that the effects of temperature are incorporated into the transformed Hamiltonian and the fermionic baths with nonzero initial temperatures are mapped to virtual fermionic baths with zero initial temperature.
In the interaction picture with respect to the environmental Hamiltonian $\mathcal{H}_{\mathrm{env}}=\sum_{\lambda k} \omega_{\lambda k}\left(d_{\lambda k}^{\dagger} d_{\lambda k}+e_{\lambda k} e_{\lambda k}^{\dagger}\right)$, the total Hamiltonian reads

$$
\begin{aligned}
\mathcal{H}(t)= & H_{\mathrm{sys}}+\sum_{\lambda k}\left(\sqrt{\bar{n}_{\lambda k}} g_{\lambda k}^{*} c_{\lambda} e_{\lambda k} e^{i \omega_{\lambda k} t}\right. \\
& \left.+\sqrt{1-\bar{n}_{\lambda k}} g_{\lambda k} c_{\lambda}^{\dagger} d_{\lambda k} e^{-i \omega_{\lambda k} t}+\text { H.c. }\right),
\end{aligned}
$$

and the quantum state of the total system satisfies the equation of motion

$$
\partial_{t}\left|\Psi_{t}\right\rangle=-i \mathcal{H}(t)\left|\Psi_{t}\right\rangle
$$

We assume that the quantum state of the total system is factorized at the initial time $t=0$, so that $\left|\Psi_{0}\right\rangle=$ $\left|\varphi_{0}\right\rangle \otimes|0\rangle$, with the virtual fermionic baths initially in the ground state (i.e., at zero temperature): $|0\rangle=\bigotimes_{\lambda}|0\rangle_{\lambda d} \otimes$ $|0\rangle_{\lambda e}$, where $d_{\lambda k}|0\rangle=0$, and $e_{\lambda k}|0\rangle=0$.

Define a femionic coherent-state basis for the environmental degrees of freedom:

$$
|z w\rangle=\bigotimes_{\lambda}|z\rangle_{\lambda} \otimes|w\rangle_{\lambda}
$$

with

$$
\begin{aligned}
|z\rangle_{\lambda} & =\bigotimes_{k}\left|z_{k}\right\rangle_{\lambda}=e^{-\sum_{k} z_{\lambda k} d_{\lambda k}^{\dagger}}|0\rangle \\
|w\rangle_{\lambda} & =\bigotimes_{k}\left|w_{k}\right\rangle_{\lambda}=e^{-\sum_{k} w_{\lambda k} e_{\lambda k}^{\dagger}}|0\rangle,
\end{aligned}
$$

where $z_{k}$ and $w_{k}$ are Grassmann variables that obey the anticommutation relation. With the completeness relation for coherent states $\int e^{-z^{*} z-w^{*} w}|z w\rangle\langle z w| d^{2} z d^{2} w=$ 1 , the state $\left|\Psi_{t}\right\rangle$ can be expressed as

$$
\left|\Psi_{t}\right\rangle=\int e^{-z^{*} z-w^{*} w}|z w\rangle \otimes\left|\psi_{t}\left(z^{*}, w^{*}\right)\right\rangle d^{2} z d^{2} w
$$

where

$$
\begin{aligned}
& z^{*} z \equiv \sum_{\lambda k} z_{\lambda k}^{*} z_{\lambda k}, \quad w^{*} w \equiv \sum_{\lambda k} w_{\lambda k}^{*} w_{\lambda k} \\
& d^{2} z \equiv \prod_{\lambda k} d z_{\lambda k}^{*} d z_{\lambda k}, \quad d^{2} w \equiv \prod_{\lambda k} d w_{\lambda k}^{*} d w_{\lambda k} .
\end{aligned}
$$

The actions of annihilation (creation) operators $d_{\lambda k}$ and $e_{\lambda k}\left(d_{\lambda k}^{\dagger}\right.$ and $\left.e_{\lambda k}^{\dagger}\right)$ on fermionic coherent states satisfy the relations [23]:

$$
\begin{aligned}
& d_{\lambda k}|z\rangle_{\lambda}=z_{\lambda k}|z\rangle_{\lambda}, \quad d_{\lambda k}^{\dagger}|z\rangle_{\lambda}=-\frac{\partial}{\partial z_{\lambda k}}|z\rangle_{\lambda}, \\
& e_{\lambda k}|w\rangle_{\lambda}=w_{\lambda k}|w\rangle_{\lambda}, \quad e_{\lambda k}^{\dagger}|w\rangle_{\lambda}=-\frac{\partial}{\partial w_{\lambda k}}|w\rangle_{\lambda} .
\end{aligned}
$$

When projecting onto the coherent-state basis, the equation of motion (7) can be reduced to the NMQSD equa- 
tion for a pure state of the system $\left|\psi_{t}\left(z^{*}, w^{*}\right)\right\rangle \equiv\left\langle z w \mid \Psi_{t}\right\rangle$ :

$$
\begin{aligned}
\frac{\partial}{\partial t}\left|\psi_{t}\right\rangle= & -i H_{\mathrm{sys}}\left|\psi_{t}\right\rangle-\sum_{\lambda}\left[c_{\lambda} z_{\lambda}^{*}(t)\left|\psi_{t}\right\rangle+c_{\lambda}^{\dagger} w_{\lambda}^{*}(t)\left|\psi_{t}\right\rangle\right] \\
& -\sum_{\lambda} c_{\lambda}^{\dagger} \int_{0}^{t} \alpha_{\lambda 1}(t-s) \frac{\delta}{\delta z_{\lambda}^{*}(s)}\left|\psi_{t}\right\rangle d s \\
& -\sum_{\lambda} c_{\lambda} \int_{0}^{t} \alpha_{\lambda 2}(t-s) \frac{\delta}{\delta w_{\lambda}^{*}(s)}\left|\psi_{t}\right\rangle d s
\end{aligned}
$$

which initiates from $\left|\psi_{t=0}\left(z^{*}, w^{*}\right)\right\rangle=\left|\varphi_{0}\right\rangle$. Here the noise functions $z_{\lambda}^{*}(t)$ and $w_{\lambda}^{*}(t)$ are defined as

$$
\begin{aligned}
& z_{\lambda}^{*}(t)=-i \sum_{k} \sqrt{1-\bar{n}_{\lambda k}} g_{\lambda k}^{*} z_{\lambda k}^{*} e^{i \omega_{\lambda k} t}, \\
& w_{\lambda}^{*}(t)=-i \sum_{k} \sqrt{\bar{n}_{\lambda k}} g_{\lambda k} w_{\lambda k}^{*} e^{-i \omega_{\lambda k} t} .
\end{aligned}
$$

The temperature-dependent environment correlation functions are

$$
\begin{aligned}
\alpha_{\lambda 1}(t-s) & \equiv \mathcal{M}\left\{z_{\lambda}(t) z_{\lambda}^{*}(s)\right\} \\
& =\int d \omega\left[1-\bar{n}_{\lambda}(\omega)\right] J_{\lambda}(\omega) e^{-i \omega(t-s)}, \\
\alpha_{\lambda 2}(t-s) & \equiv \mathcal{M}\left\{w_{\lambda}(t) w_{\lambda}^{*}(s)\right\} \\
& =\int d \omega \bar{n}_{\lambda}(\omega) J_{\lambda}(\omega) e^{i \omega(t-s)},
\end{aligned}
$$

where $\mathcal{M}\{\cdot\}$ denotes the statistical mean over all noise variables: $\mathcal{M}\{\cdot\} \equiv \int e^{-z^{*} z-w^{*} w}\{\cdot\} d^{2} z d^{2} w$.

Introducing $O$-operators by

$$
\begin{aligned}
\frac{\delta}{\delta z_{\lambda}^{*}(s)}\left|\psi_{t}\left(z^{*}, w^{*}\right)\right\rangle & =O_{\lambda 1}\left(t, s, z^{*}, w^{*}\right)\left|\psi_{t}\left(z^{*}, w^{*}\right)\right\rangle, \\
\frac{\delta}{\delta w_{\lambda}^{*}(s)}\left|\psi_{t}\left(z^{*}, w^{*}\right)\right\rangle & =O_{\lambda 2}\left(t, s, z^{*}, w^{*}\right)\left|\psi_{t}\left(z^{*}, w^{*}\right)\right\rangle,
\end{aligned}
$$

we can write the NMQSD equation in a time-local form:

$$
\begin{aligned}
\frac{\partial}{\partial t}\left|\psi_{t}\right\rangle= & -i H_{\mathrm{sys}}\left|\psi_{t}\right\rangle-\sum_{\lambda}\left[c_{\lambda} z_{\lambda}^{*}(t)+c_{\lambda}^{\dagger} w_{\lambda}^{*}(t)\right. \\
& \left.+c_{\lambda}^{\dagger} \bar{O}_{\lambda 1}\left(t, z^{*}, w^{*}\right)+c_{\lambda} \bar{O}_{\lambda 2}\left(t, z^{*}, w^{*}\right)\right]\left|\psi_{t}\right\rangle
\end{aligned}
$$

where $\bar{O}_{\lambda n} \equiv \int_{0}^{t} d s \alpha_{\lambda n}(t-s) O_{\lambda n}\left(t, s, z^{*}, w^{*}\right), n=1,2$.

With the consistency conditions

$$
\frac{\partial}{\partial t} \frac{\delta\left|\psi_{t}\right\rangle}{\delta z_{\lambda}^{*}(s)}=\frac{\delta}{\delta z_{\lambda}^{*}(s)} \frac{\partial\left|\psi_{t}\right\rangle}{\partial t}, \quad \frac{\partial}{\partial t} \frac{\delta\left|\psi_{t}\right\rangle}{\delta w_{\lambda}^{*}(s)}=\frac{\delta}{\delta w_{\lambda}^{*}(s)} \frac{\partial\left|\psi_{t}\right\rangle}{\partial t}
$$

as well as the initial conditions $\left.O_{\lambda 1}\left(t, s, z^{*}, w^{*}\right)\right|_{t=s}=c_{\lambda}$ and $\left.O_{\lambda 2}\left(t, s, z^{*}, w^{*}\right)\right|_{t=s}=c_{\lambda}^{\dagger}$, we obtain the equations of motion for the $O$-operators:

$$
\begin{aligned}
\frac{\partial O_{\lambda n}}{\partial t}= & {\left[-i H_{\mathrm{sys}}-\sum_{\lambda^{\prime}}\left(c_{\lambda^{\prime}}^{\dagger} \bar{O}_{\lambda^{\prime} 1}+c_{\lambda^{\prime}} \bar{O}_{\lambda^{\prime} 2}\right), O_{\lambda n}\right]+Q_{n} } \\
& +\sum_{\lambda^{\prime}}\left(\left\{c_{\lambda^{\prime}}, O_{\lambda n}\right\} z_{\lambda^{\prime}}^{*}(t)+\left\{c_{\lambda^{\prime}}^{\dagger}, O_{\lambda n}\right\} w_{\lambda^{\prime}}^{*}(t)\right),
\end{aligned}
$$

where the square and curly brackets denote the commutator and anticommutator, respectively, and

$$
Q_{n}=c_{L}^{\dagger} \frac{\delta \bar{O}_{L 1}}{\delta \Lambda_{n}}+c_{R}^{\dagger} \frac{\delta \bar{O}_{R 1}}{\delta \Lambda_{n}}+c_{L} \frac{\delta \bar{O}_{L 2}}{\delta \Lambda_{n}}+c_{R} \frac{\delta \bar{O}_{R 2}}{\delta \Lambda_{n}},
$$

with $\Lambda_{1}=z_{\lambda}^{*}(s)$, and $\Lambda_{2}=w_{\lambda}^{*}(s)$.

\section{MASTER EQUATION}

The reduced density operator of an open quantum system by tracing over the environmental degrees of freedom can be obtained by taking the statistical mean for a density operator related to the state $\left|\psi_{t}\left(z^{*}, w^{*}\right)\right\rangle$ :

$$
\rho_{t}=\operatorname{Tr}_{\mathrm{env}}\left|\Psi_{t}\right\rangle\left\langle\Psi_{t}\right|=\mathcal{M}\left\{P_{t}\right\},
$$

where $P_{t} \equiv\left|\psi_{t}\left(z^{*}, w^{*}\right)\right\rangle\left\langle\psi_{t}(-z,-w)\right|$.

Using the relation

$$
\begin{aligned}
\frac{\partial P_{t}}{\partial t}= & \frac{\partial\left|\psi_{t}\left(z^{*}, w^{*}\right)\right\rangle}{\partial t}\left\langle\psi_{t}(-z,-w)\right| \\
& +\left|\psi_{t}\left(z^{*}, w^{*}\right)\right\rangle \frac{\partial\left\langle\psi_{t}(-z,-w)\right|}{\partial t},
\end{aligned}
$$

and Eq. (17), we derive the following non-Markovian master equation:

$$
\begin{aligned}
\frac{\partial \rho_{t}}{\partial t}= & -i\left[H_{\mathrm{sys}}, \rho_{t}\right]+\sum_{\lambda}\left(\left[c_{\lambda}, \mathcal{M}\left\{P_{t} \bar{O}_{\lambda 1}^{\dagger}(t,-z,-w)\right\}\right]\right. \\
& -\left[c_{\lambda}^{\dagger}, \mathcal{M}\left\{\bar{O}_{\lambda 1}\left(t, z^{*}, w^{*}\right) P_{t}\right\}\right] \\
& -\left[c_{\lambda}, \mathcal{M}\left\{\bar{O}_{\lambda 2}\left(t, z^{*}, w^{*}\right) P_{t}\right\}\right] \\
& \left.+\left[c_{\lambda}^{\dagger}, \mathcal{M}\left\{P_{t} \bar{O}_{\lambda 2}^{\dagger}(t,-z,-w)\right\}\right]\right)
\end{aligned}
$$

This master equation is derived non-perturbatively, so it applies even for a strong coupling between the system and the environments. Moreover, in addition to trace preserving, it also preserves the positivity and hermiticity.

In the Markovian limit, there are

$$
\begin{aligned}
& \alpha_{\lambda 1}(t-s) \rightarrow\left(1-\bar{n}_{\lambda}\right) \Gamma_{\lambda} \delta(t-s), \\
& \alpha_{\lambda 2}(t-s) \rightarrow \bar{n}_{\lambda} \Gamma_{\lambda} \delta(t-s),
\end{aligned}
$$

where $\Gamma_{\lambda}=2 \pi \rho_{\lambda}\left|g_{\lambda}\right|^{2}$, with $\lambda=L(R)$, is the electron tunneling rate between the system and the left (right) lead. Also, the time-integrated $O$-operators become

$$
\begin{aligned}
& \bar{O}_{\lambda 1} \rightarrow \frac{1}{2} \Gamma_{\lambda}\left(1-\bar{n}_{\lambda}\right) c_{\lambda}, \\
& \bar{O}_{\lambda 2} \rightarrow \frac{1}{2} \Gamma_{\lambda} \bar{n}_{\lambda} c_{\lambda}^{\dagger} .
\end{aligned}
$$

Therefore, the master equation (23) is reduced to

$$
\begin{aligned}
\frac{\partial \rho_{t}}{\partial t}= & -i\left[H_{\mathrm{sys}}, \rho_{t}\right] \\
& +\sum_{\lambda} \frac{\Gamma_{\lambda}}{2}\left[\bar{n}_{\lambda}\left(2 c_{\lambda}^{\dagger} \rho_{t} c_{\lambda}-c_{\lambda} c_{\lambda}^{\dagger} \rho_{t}-\rho_{t} c_{\lambda} c_{\lambda}^{\dagger}\right)\right. \\
& \left.+\left(1-\bar{n}_{\lambda}\right)\left(2 c_{\lambda} \rho_{t} c_{\lambda}^{\dagger}-c_{\lambda}^{\dagger} c_{\lambda} \rho_{t}-\rho_{t} c_{\lambda}^{\dagger} c_{\lambda}\right)\right] .
\end{aligned}
$$


It is clear that this Markov master equation has a Lindblad form.

\section{APPLICATION TO QUANTUM-DOT SYSTEMS}

Below we apply our NMQSD approach to single and double quantun-dot systems.

\section{A. Single quantum dot}

Suppose that the single quantum dot is in the strong Coulomb blockade regime, so that only one electron is allowed therein. The Hamiltonian of the system is written as

$$
H_{\mathrm{sys}}=\omega_{0} c^{\dagger} c
$$

and $c_{L}=c_{R}=c$ for $H_{\text {int }}$ in Eq. (2). The non-Markovian master equation is exactly derived as (see Appendix A)

$$
\begin{aligned}
\frac{\partial \rho_{t}}{\partial t}= & -i\left[H_{\mathrm{sys}}, \rho_{t}\right]+\Gamma_{1}(t)\left[c, \rho_{t} c^{\dagger}\right]+\Gamma_{2}(t)\left[c, c^{\dagger} \rho_{t}\right] \\
& +\Gamma_{1}^{*}(t)\left[c \rho_{t}, c^{\dagger}\right]+\Gamma_{2}^{*}(t)\left[\rho_{t} c, c^{\dagger}\right]
\end{aligned}
$$

with time-dependent rates

$$
\Gamma_{j}(t)=\int_{0}^{t}\left[\alpha_{1}(s-t) A_{j}(t, s)-\alpha_{2}(t-s) B_{j}(t, s)\right] d s
$$

where $\alpha_{j}(t)=\alpha_{L j}(t)+\alpha_{R j}(t) ; A_{j}(t, s)$ and $B_{j}(t, s)$ are determined by the integro-differential equations:

$$
\begin{aligned}
& \left(\frac{\partial}{\partial s}-i \omega_{0}\right) A_{j}(t, s)+\int_{0}^{s} \beta\left(s-s^{\prime}\right) A_{j}\left(t, s^{\prime}\right) d s^{\prime}=U(t, s) \\
& \left(\frac{\partial}{\partial s}-i \omega_{0}\right) B_{j}(t, s)+\int_{0}^{s} \beta\left(s-s^{\prime}\right) B_{j}\left(t, s^{\prime}\right) d s^{\prime}=V(t, s),
\end{aligned}
$$

with

$$
\begin{aligned}
& \beta\left(s-s^{\prime}\right) \equiv \alpha_{1}\left(s^{\prime}-s\right)+\alpha_{2}\left(s-s^{\prime}\right) \\
& U(t, s) \equiv \int_{0}^{t} \alpha_{2}\left(s-s^{\prime}\right) h\left(t, s^{\prime}\right) d s^{\prime} \\
& V(t, s) \equiv \int_{0}^{t} \alpha_{1}\left(s^{\prime}-s\right) h\left(t, s^{\prime}\right) d s^{\prime}
\end{aligned}
$$

and the final conditions at $s=t: A_{1}(t, t)=B_{1}(t, t)=1$, and $A_{2}(t, t)=B_{2}(t, t)=0$. Here $h(t, s)$ satisfies the equation

$$
\left(\frac{\partial}{\partial s}-i \omega_{0}\right) h(t, s)-\int_{s}^{t} \beta\left(s-s^{\prime}\right) h\left(t, s^{\prime}\right) d s^{\prime}=0,
$$

with the final condition $h(t, t)=1$.
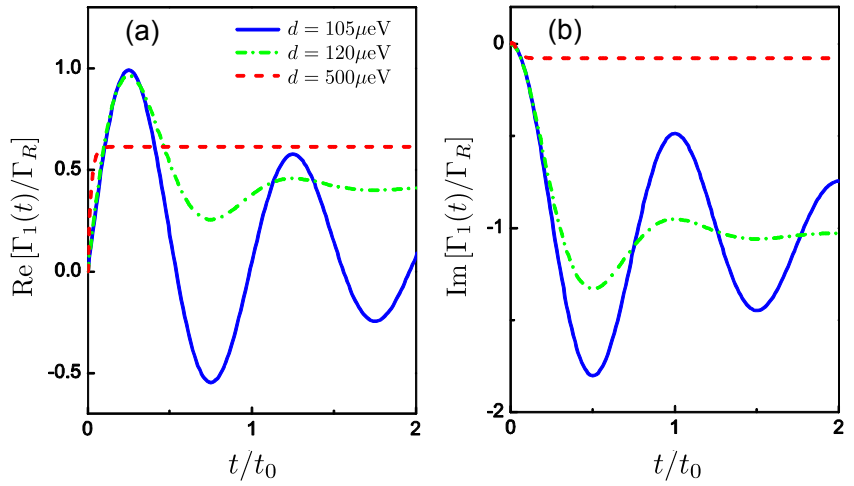

FIG. 1: (Color online) (a) Real and (b) imaginary parts of the time-dependent coefficient $\Gamma_{1}(t)$ in the non-Markovian master equation of a single quantum dot system, where $\Gamma_{L}=\Gamma_{R}=$ $100 \mu \mathrm{eV}, \omega_{0}=50 \mu \mathrm{eV}$, and $t_{0}=2 \pi / \omega_{0}$.

In the Markovian limit, there are

$$
\begin{aligned}
& \Gamma_{1}(t) \rightarrow \frac{1}{2}\left[1-\bar{n}_{L}\left(\omega_{0}\right)\right] \Gamma_{L}+\frac{1}{2}\left[1-\bar{n}_{R}\left(\omega_{0}\right)\right] \Gamma_{R}, \\
& \Gamma_{2}(t) \rightarrow-\frac{1}{2} \bar{n}_{L}\left(\omega_{0}\right) \Gamma_{L}-\frac{1}{2} \bar{n}_{R}\left(\omega_{0}\right) \Gamma_{R} .
\end{aligned}
$$

Let us consider the zero-temperature case with $\bar{n}_{\lambda}\left(\omega_{0}\right) \rightarrow$ $\theta\left(\mu_{\lambda}-\omega_{0}\right)$, were $\theta$ is the Heaviside step function. If the single-dot level $\omega_{0}$ lies within the energy window $\mu_{L}>$ $\omega_{0}>\mu_{R}$, the master equation (28) reduces to

$$
\begin{aligned}
\frac{\partial}{\partial t} \rho_{t}= & -i \omega_{0}\left[c^{\dagger} c, \rho_{t}\right]+\frac{1}{2} \Gamma_{L}\left(2 c^{\dagger} \rho_{t} c-c c^{\dagger} \rho_{t}-\rho_{t} c c^{\dagger}\right) \\
& +\frac{1}{2} \Gamma_{R}\left(2 c \rho_{t} c^{\dagger}-c^{\dagger} c \rho_{t}-\rho_{t} c^{\dagger} c\right) .
\end{aligned}
$$

With the basis state $|0\rangle(|1\rangle)$ which denotes an empty (occupied) dot, it follows that the density matrix elements satisfy

$$
\begin{aligned}
& \dot{\rho}_{00}=-\Gamma_{L} \rho_{00}+\Gamma_{R} \rho_{11}, \\
& \dot{\rho}_{11}=\Gamma_{L} \rho_{00}-\Gamma_{R} \rho_{11}, \\
& \dot{\rho}_{10}=-\left(i \omega_{0}+\Gamma_{L}+\Gamma_{R}\right) \rho_{10},
\end{aligned}
$$

which are exactly the rate equations obtained by Gurvitz and Prager [14].

Figure 1 presents both real and imaginary parts of the time-dependent coefficients $\Gamma_{1}(t)$ in Eq. (28). For simplicity, we consider the large bias regime (i.e., $\mu_{L} \gg$ $\left.\omega_{0} \gg \mu_{R}\right)$ and a zero temperature for fermionic environments. The tunneling rates are chosen in the symmetric case of $\Gamma_{L}=\Gamma_{R}=\Gamma$, so that $\Gamma_{2}(t)=-\Gamma_{1}(t)$. Moreover, the noise is modeled as the Ornstein-Uhlenbeck process, and the related correlation functions are then given by

$$
\begin{aligned}
& \alpha_{L 2}(t, s)=\alpha_{R 1}(t, s)=\frac{\Gamma d}{2} e^{-d|t-s|}, \\
& \alpha_{L 1}(t, s)=\alpha_{R 2}(t, s)=0,
\end{aligned}
$$

where $1 / d$ characterizes the memory time of each environment. In the Markovian limit with $d \rightarrow \infty, \alpha_{L 2}(t, s)=$ 
$\alpha_{R 1}(t, s) \rightarrow \Gamma \delta(t-s)$, and $\Gamma_{1}(t)$ becomes time-dependent. Indeed, Fig. 1 shows that $\Gamma_{1}$ oscillates with time $t$, but it quickly decays to a constant for a large value of $d$.

\section{B. Double quantum dot (DQD)}

Suppose that the DQD is in the strong Coulomb blockade regime, so that at most one electron is allowed in each dot. The Hamiltonian of the DQD can be written as

$$
H_{\mathrm{sys}}=\omega_{1} c_{1}^{\dagger} c_{1}+\omega_{2} c_{2}^{\dagger} c_{2}+\Omega_{0}\left(c_{2}^{\dagger} c_{1}+c_{1}^{\dagger} c_{2}\right)
$$

where $\Omega_{0}$ denotes the inderdot coupling. For $H_{\text {int }}$ in Eq. (2), $c_{L}=c_{1}$, and $c_{R}=c_{2}$. The exact non-Markovian master equation is given by

$$
\begin{aligned}
\frac{\partial \rho_{t}}{\partial t}= & -i\left[H_{\mathrm{sys}}, \rho_{t}\right]+\left\{\left(\Gamma_{L 1}(t)\left[c_{1}, \rho_{t} c_{1}^{\dagger}\right]+\Gamma_{L 2}(t)\left[c_{1}, c_{1}^{\dagger} \rho_{t}\right]\right.\right. \\
& +\Gamma_{L 3}(t)\left[c_{1}, \rho_{t} c_{2}^{\dagger}\right]+\Gamma_{L 4}(t)\left[c_{1}, c_{2}^{\dagger} \rho_{t}\right] \\
& +\Gamma_{R 1}(t)\left[c_{2}, \rho_{t} c_{1}^{\dagger}\right]+\Gamma_{R 2}(t)\left[c_{2}, c_{1}^{\dagger} \rho_{t}\right] \\
& \left.\left.+\Gamma_{R 3}(t)\left[c_{2}, \rho_{t} c_{2}^{\dagger}\right]+\Gamma_{R 4}(t)\left[c_{2}, c_{2}^{\dagger} \rho_{t}\right]\right)+ \text { H.c. }\right\},
\end{aligned}
$$

with time-dependent coefficients

$\Gamma_{\lambda j}(t)=\int_{0}^{t}\left[\alpha_{\lambda 1}(s-t) A_{\lambda j}(t, s)-\alpha_{\lambda 2}(t-s) B_{\lambda j}(t, s)\right] d s$,

where $A_{\lambda j}(t, s)$ and $B_{\lambda j}(t, s)$ satisfy a set of integrodifferential equations (see Appendix B), with the final conditions: $A_{L 1}(t, t)=A_{R 3}(t, t)=B_{L 2}(t, t)=$ $B_{R 4}(t, t)=1$, and $A_{\lambda j}(t, t)=0, B_{\lambda j}(t, t)=0$ for other $\lambda$ and $j$. A similar non-Markovian master equation was also obtained using the Feynman-Vernon influence functional path-integral method [12].

In the Markovian limit, there are

$$
\begin{aligned}
& \Gamma_{L 1}(t) \rightarrow \frac{1}{2}\left[1-\bar{n}_{L}\left(\omega_{1}\right)\right] \Gamma_{L}, \quad \Gamma_{R 1} \rightarrow 0 ; \\
& \Gamma_{L 2}(t) \rightarrow-\frac{1}{2} \bar{n}_{L}\left(\omega_{1}\right) \Gamma_{L}, \quad \Gamma_{R 2} \rightarrow 0 ; \\
& \Gamma_{L 3}(t) \rightarrow 0, \quad \Gamma_{R 3} \rightarrow \frac{1}{2}\left[1-\bar{n}_{R}\left(\omega_{2}\right)\right] \Gamma_{R} ; \\
& \left.\Gamma_{L 4}(t) \rightarrow 0, \quad \Gamma_{R 4} \rightarrow-\frac{1}{2} \bar{n}_{R}\left(\omega_{2}\right)\right] \Gamma_{R} .
\end{aligned}
$$

We also consider the zero-temperature case with $\bar{n}_{\lambda}\left(\omega_{n}\right) \rightarrow \theta\left(\mu_{\lambda}-\omega_{n}\right)$, and the two single-dot levels of the DQD all lie within the energy window $\mu_{L}>\omega_{n}>\mu_{R}$, where $n=1,2$. We use $|l\rangle, l=0,1,2$ and 3 , to denote the states with both dots empty, the left dot occupied, the right dot occupied, and both dots occupied, respectively. From Eq. (38), it follows that the master equations for density matrix elements are reduced to

$$
\begin{aligned}
& \dot{\rho}_{00}=-\Gamma_{L} \rho_{00}+\Gamma_{R} \rho_{22}, \quad \dot{\rho}_{33}=\Gamma_{L} \rho_{22}-\Gamma_{R} \rho_{33}, \\
& \dot{\rho}_{11}=\Gamma_{L} \rho_{00}+\Gamma_{R} \rho_{33}+i \Omega_{0}\left(\rho_{12}-\rho_{21}\right), \\
& \dot{\rho}_{22}=-\left(\Gamma_{L}+\Gamma_{R}\right) \rho_{22}-i \Omega_{0}\left(\rho_{12}-\rho_{21}\right), \\
& \dot{\rho}_{12}=-i\left(\omega_{1}-\omega_{2}\right) \rho_{12}+i \Omega_{0}\left(\rho_{11}-\rho_{22}\right)-\frac{\Gamma_{L}+\Gamma_{R}}{2} \rho_{12},
\end{aligned}
$$

which are identical to the rate equations obtained in [14]. For a DQD, both intradot and interdot Coulomb repulsions can play an important role in the Coulomb-blockade effect (see, e.g., 24]). Thus, if both intradot and interdot Coulomb repulsions are so strong that only one electron is allowed in the whole DQD, the master equations for density matrix elements are reduced to

$$
\begin{aligned}
& \dot{\rho}_{00}=-\Gamma_{L} \rho_{00}+\Gamma_{R} \rho_{22} \\
& \dot{\rho}_{11}=\Gamma_{L} \rho_{00}+i \Omega_{0}\left(\rho_{12}-\rho_{21}\right) \\
& \dot{\rho}_{22}=-\Gamma_{R} \rho_{22}-i \Omega_{0}\left(\rho_{12}-\rho_{21}\right) \\
& \dot{\rho}_{12}=-i\left(\omega_{1}-\omega_{2}\right) \rho_{12}+i \Omega_{0}\left(\rho_{11}-\rho_{22}\right)-\frac{\Gamma_{R}}{2} \rho_{12},
\end{aligned}
$$

which are exactly the rate equations obtained in [15].

\section{CONCLUSION}

We have developed an NMQSD method to study the dynamics of an open quantum system in fermionic baths. We not only obtain the NMQSD equation for quantum states of the system, but also derive the non-Markovian master equation. This non-Markovian approach is formulated in a non-perturbative manner and it applies even for a strong coupling between the system and the fermionic baths. Moreover, as useful examples, we have applied this NMQSD method to single and double quantum-dot systems.

Note added: After finishing this work, we were aware of a closely related work in [25], which also investigated an open quantum system in fermionic baths by using a quantum state diffusion approach.

\section{Acknowledgments}

This work was supported by the National Basic Research Program of China Grant No. 2009CB929302 and the National Natural Science Foundation of China Grant No. 91121015.

\section{Appendix A: Non-Markovian master equation for a single quantum dot sytem}

In this appendix, we show how to obtain the exact non-Markovian master equation for a single quantum 
dot system from the general non-Markovian master equation (23) in the main text. In order to get a closed convolutionless master equation, we need to know the explicit forms of the operator functions

$$
\begin{aligned}
& \mathcal{Q}_{1}(t, s) \equiv \mathcal{M}\left\{O_{\lambda 1}\left(t, s, z^{*}, w^{*}\right) P_{t}\right\}, \\
& \mathcal{Q}_{2}(t, s) \equiv \mathcal{M}\left\{O_{\lambda 2}\left(t, s, z^{*}, w^{*}\right) P_{t}\right\},
\end{aligned}
$$

and their hermitian conjugates. Similar to the Heisenberg equation approach for quantum state diffusion in a bosonic environment [7, 22], we start to obtain the evolution equations for

$$
\begin{aligned}
& \mathcal{C}_{1}(s)=\left\langle z w\left|\mathcal{U}_{t} c(s)\right| 0\right\rangle=O_{\lambda 1}\left(t, s, z^{*}, w^{*}\right) G_{t}\left(z^{*}, w^{*}\right), \\
& \mathcal{C}_{2}(s)=\left\langle z w\left|\mathcal{U}_{t} c^{\dagger}(s)\right| 0\right\rangle=O_{\lambda 2}\left(t, s, z^{*}, w^{*}\right) G_{t}\left(z^{*}, w^{*}\right)
\end{aligned}
$$

where $\mathcal{U}_{t}$ is the time evolution operator for the total system: $\left|\Psi_{t}\right\rangle=\mathcal{U}_{t}\left|\Psi_{0}\right\rangle$. The stochastic propagator of the open quantum system can be expressed using the matrix element of the time evolution operator: $G_{t}\left(z^{*}, w^{*}\right)=$ $\left\langle z w\left|\mathcal{U}_{t}\right| 0\right\rangle$. Now we take the operators $\mathcal{C}_{i}(i=1,2)$ as functions of $s$ and treat $t$ only as a parameter. With the Heisenberg equation of motion for the fermionic operator $c(s)$ of the single quantum dot:

$$
\frac{\partial}{\partial s} c(s)=-i \mathcal{U}_{s}^{-1}[c, \mathcal{H}(s)] \mathcal{U}_{s},
$$

we have

$$
\begin{aligned}
\frac{\partial}{\partial s} \mathcal{C}_{1}(s)= & -i \omega_{0} \mathcal{C}_{1}(s) \\
& -i \sum_{\lambda k} \sqrt{1-\bar{n}_{\lambda k}} g_{\lambda k} e^{-i \omega_{\lambda k} s}\left\langle z w\left|\mathcal{U}_{t} d_{\lambda k}(s)\right| 0\right\rangle \\
& +i \sum_{\lambda k} \sqrt{\bar{n}_{\lambda k}} g_{\lambda k} e^{-i \omega_{\lambda k} s}\left\langle z w\left|\mathcal{U}_{t} e_{\lambda k}^{\dagger}(s)\right| 0\right\rangle
\end{aligned}
$$

By integrating the Heisenberg equations of motion for both $d_{\lambda k}$ and $e_{\lambda k}^{\dagger}$, we can get

$$
\begin{aligned}
& d_{\lambda k}(s)=d_{\lambda k}-i \sqrt{1-\bar{n}_{\lambda k}} g_{\lambda k}^{*} \int_{0}^{s} e^{i \omega_{\lambda k} s^{\prime}} c\left(s^{\prime}\right) d s^{\prime}, \\
& e_{\lambda k}^{\dagger}(t)=e_{\lambda k}^{\dagger}(s)+i \sqrt{\bar{n}_{\lambda k}} g_{\lambda k}^{*} \int_{s}^{t} e^{i \omega_{\lambda k} s^{\prime}} c\left(s^{\prime}\right) d s^{\prime} .
\end{aligned}
$$

Substituting Eq. (A5) into Eq. (A4), we derive the evolution equation for $\mathcal{C}_{1}(s)$ as

$$
\begin{aligned}
\frac{\partial}{\partial s} \mathcal{C}_{1}(s)= & -i \omega_{0} \mathcal{C}_{1}(s) \\
& -\int_{0}^{s}\left[\alpha_{L 1}\left(s-s^{\prime}\right)+\alpha_{R 1}\left(s-s^{\prime}\right)\right] \mathcal{C}_{1}\left(s^{\prime}\right) d s^{\prime} \\
& +\int_{s}^{t}\left[\alpha_{L 2}\left(s^{\prime}-s\right)+\alpha_{R 2}\left(s^{\prime}-s\right)\right] \mathcal{C}_{1}\left(s^{\prime}\right) d s^{\prime} \\
& -\left[w_{L}^{*}(s)+w_{R}^{*}(s)\right]\langle z w|\mathcal{U}(t)| 0\rangle .
\end{aligned}
$$

In the above calculations, we have used the relations $d_{\lambda k}|0\rangle=0$, and $\left\langle z w\left|\mathcal{U}_{t} e_{\lambda k}^{\dagger}(t)\right| 0\right\rangle=w_{\lambda k}^{*}\left\langle z w\left|\mathcal{U}_{t}\right| 0\right\rangle$. Similarly, we can also derive the evolution equation for $\mathcal{C}_{2}(s)$ as

$$
\begin{aligned}
\frac{\partial}{\partial s} \mathcal{C}_{2}(s)= & i \omega_{0} \mathcal{C}_{2}(s) \\
& +\int_{s}^{t}\left[\alpha_{L 1}\left(s^{\prime}-s\right)+\alpha_{R 1}\left(s^{\prime}-s\right)\right] \mathcal{C}_{2}\left(s^{\prime}\right) d s^{\prime} \\
& -\int_{0}^{s}\left[\alpha_{L 2}\left(s-s^{\prime}\right)+\alpha_{R 2}\left(s-s^{\prime}\right)\right] \mathcal{C}_{2}\left(s^{\prime}\right) d s^{\prime} \\
& -\left[z_{L}^{*}(s)+z_{R}^{*}(s)\right]\langle z w|\mathcal{U}(t)| 0\rangle .
\end{aligned}
$$

From Eq. (A2), we can thus obtain the evolution equation for the $O$-operators $O_{\lambda 1}\left(t, s, z^{*}, w^{*}\right)$ and $O_{\lambda 2}\left(t, s, z^{*}, w^{*}\right)$ :

$$
\begin{aligned}
& \frac{\partial}{\partial s} O_{\lambda 1}\left(t, s, z^{*}, w^{*}\right)=-i \omega_{0} O_{\lambda 1}\left(t, s, z^{*}, w^{*}\right) \\
& -\int_{0}^{s}\left[\alpha_{L 1}\left(s-s^{\prime}\right)+\alpha_{R 1}\left(s-s^{\prime}\right)\right] O_{\lambda 1}\left(t, s^{\prime}, z^{*}, w^{*}\right) d s^{\prime} \\
& +\int_{s}^{t}\left[\alpha_{L 2}\left(s^{\prime}-s\right)+\alpha_{R 2}\left(s^{\prime}-s\right)\right] O_{\lambda 1}\left(t, s^{\prime}, z^{*}, w^{*}\right) d s^{\prime} \\
& -\left[w_{L}^{*}(s)+w_{R}^{*}(s)\right],
\end{aligned}
$$

and

$$
\begin{aligned}
& \frac{\partial}{\partial s} O_{\lambda 2}\left(t, s, z^{*}, w^{*}\right)=i \omega_{0} O_{\lambda 2}\left(t, s, z^{*}, w^{*}\right) \\
& +\int_{s}^{t}\left[\alpha_{L 1}\left(s^{\prime}-s\right)+\alpha_{R 1}\left(s^{\prime}-s\right)\right] O_{\lambda 2}\left(t, s^{\prime}, z^{*}, w^{*}\right) d s^{\prime} \\
& -\int_{0}^{s}\left[\alpha_{L 2}\left(s-s^{\prime}\right)+\alpha_{R 2}\left(s-s^{\prime}\right)\right] O_{\lambda 2}\left(t, s^{\prime}, z^{*}, w^{*}\right) d s^{\prime} \\
& -\left[z_{L}^{*}(s)+z_{R}^{*}(s)\right],
\end{aligned}
$$

with final conditions at $s=t$ :

$$
O_{\lambda 1}\left(t, t, z^{*}, w^{*}\right)=c, \quad O_{\lambda 2}\left(t, t, z^{*}, w^{*}\right)=c^{\dagger} .
$$

By taking the mean $\mathcal{M}\left\{\cdots P_{t}\right\}$ on Eqs. (A8) and (A9), and using the relations

$$
\begin{aligned}
& \mathcal{M}\left\{w_{\lambda}^{*}(s) P_{t}\right\}=-\mathcal{M}\left\{P_{t} \bar{O}_{\lambda 2}^{\dagger}(t,-z,-w)\right\} \\
& \mathcal{M}\left\{z_{\lambda}^{*}(s) P_{t}\right\}=-\mathcal{M}\left\{P_{t} \bar{O}_{\lambda 1}^{\dagger}(t,-z,-w)\right\}
\end{aligned}
$$

we derive the evolution equations for the operator functions $\mathcal{Q}_{n}(s)(n=1,2)$ as

$$
\begin{aligned}
& \frac{\partial}{\partial s} \mathcal{Q}_{1}(t, s)=-i \omega_{0} \mathcal{Q}_{1}(t, s) \\
& -\int_{0}^{s}\left[\alpha_{L 1}\left(s-s^{\prime}\right)+\alpha_{R 1}\left(s-s^{\prime}\right)\right] \mathcal{Q}_{1}\left(t, s^{\prime}\right) d s^{\prime} \\
& +\int_{s}^{t}\left[\alpha_{L 2}\left(s^{\prime}-s\right)+\alpha_{R 2}\left(s^{\prime}-s\right)\right] \mathcal{Q}_{1}\left(t, s^{\prime}\right) d s^{\prime} \\
& +\int_{0}^{t}\left[\alpha_{L 2}^{*}\left(s-s^{\prime}\right)+\alpha_{R 2}^{*}\left(s-s^{\prime}\right)\right] \mathcal{Q}_{2}^{\dagger}\left(t, s^{\prime}\right) d s^{\prime},
\end{aligned}
$$


and

$$
\begin{aligned}
& \frac{\partial}{\partial s} \mathcal{Q}_{2}(t, s)=i \omega_{0} \mathcal{Q}_{2}(t, s) \\
& +\int_{s}^{t}\left[\alpha_{L 1}\left(s^{\prime}-s\right)+\alpha_{R 1}\left(s^{\prime}-s\right)\right] \mathcal{Q}_{2}\left(t, s^{\prime}\right) d s^{\prime} \\
& -\int_{0}^{s}\left[\alpha_{L 2}\left(s-s^{\prime}\right)+\alpha_{R 2}\left(s-s^{\prime}\right)\right] \mathcal{R}_{2}\left(t, s^{\prime}\right) d s^{\prime} \\
& +\int_{0}^{t}\left[\alpha_{L 1}^{*}\left(s-s^{\prime}\right)+\alpha_{R 1}^{*}\left(s-s^{\prime}\right)\right] \mathcal{Q}_{1}^{\dagger}\left(t, s^{\prime}\right) d s^{\prime},
\end{aligned}
$$

with final conditions at $s=t: \mathcal{Q}_{1}(t, t)=c \rho_{t}$, and $\mathcal{Q}_{2}(t, t)=c^{\dagger} \rho_{t}$.

According to Eqs. (A12) and A13) and the final conditions of the operator functions $\mathcal{Q}_{n}(t, s), n=1,2$, it can be seen that $\mathcal{Q}_{1}(t, s)$ and $\mathcal{Q}_{2}(t, s)$ should have the forms

$$
\begin{aligned}
& \mathcal{Q}_{1}(t, s)=A_{1}^{*}(t, s) c \rho_{t}+A_{2}^{*}(t, s) \rho_{t} c, \\
& \mathcal{Q}_{2}(t, s)=B_{1}(t, s) c^{\dagger} \rho_{t}+B_{2}(t, s) \rho_{t} c^{\dagger} .
\end{aligned}
$$

Substituting $\mathcal{Q}_{1}(t, s)$ and $\mathcal{Q}_{2}(t, s)$ and their hermitian conjugates into Eq. (23), we finally obtain the nonMarkovian master equation for a single quantum dot system

$$
\begin{aligned}
\frac{\partial}{\partial t} \rho_{t}= & -i \omega_{0}\left[c^{\dagger} c, \rho_{t}\right]+\Gamma_{1}(t)\left[c, \rho_{t} c^{\dagger}\right]+\Gamma_{2}(t)\left[c, c^{\dagger} \rho_{t}\right] \\
& +\Gamma_{1}^{*}(t)\left[c \rho_{t}, c^{\dagger}\right]+\Gamma_{2}^{*}(t)\left[\rho_{t} c, c^{\dagger}\right],
\end{aligned}
$$

which is just Eq. (28) in the main text. Here the timedependent coefficients $\Gamma_{j}(t), j=1$ and 2 , are given in Eq. (29).

\section{Appendix B: Non-Markovian master equation for a double quantum dot system}

As in Appendix A, by the Heisenberg equation approach, it can be derived for the double quantum dot system that the $O$-operators satisfy the following integrodifferential equations:

$$
\begin{aligned}
& \frac{\partial}{\partial s} O_{L 1}\left(t, s, z^{*}, w^{*}\right)=-i \omega_{1} O_{L 1}\left(t, s, z^{*}, w^{*}\right) \\
& \quad-i \Omega_{0} O_{R 1}\left(t, s, z^{*}, w^{*}\right) \\
& \quad-\int_{0}^{s} \alpha_{L 1}\left(s-s^{\prime}\right) O_{L 1}\left(t, s^{\prime}, z^{*}, w^{*}\right) d s^{\prime} \\
& \quad+\int_{s}^{t} \alpha_{L 2}\left(s^{\prime}-s\right) O_{L 1}\left(t, s^{\prime}, z^{*}, w^{*}\right) d s^{\prime}-w_{L}^{*}(s), \\
& \frac{\partial}{\partial s} O_{R 1}\left(t, s, z^{*}, w^{*}\right)=-i \omega_{2} O_{R 1}\left(t, s, z^{*}, w^{*}\right) \\
& \quad-i \Omega_{0} O_{L 1}\left(t, s, z^{*}, w^{*}\right) \\
& \quad-\int_{0}^{s} \alpha_{R 1}\left(s-s^{\prime}\right) O_{R 1}\left(t, s^{\prime}, z^{*}, w^{*}\right) d s^{\prime} \\
& \quad+\int_{s}^{t} \alpha_{R 2}\left(s^{\prime}-s\right) O_{R 1}\left(t, s^{\prime}, z^{*}, w^{*}\right) d s^{\prime}-w_{R}^{*}(s),
\end{aligned}
$$

and

$$
\begin{aligned}
& \frac{\partial}{\partial s} O_{L 2}\left(t, s, z^{*}, w^{*}\right)=i \omega_{1} O_{L 2}\left(t, s, z^{*}, w^{*}\right) \\
& \quad+i \Omega_{0} O_{R 2}\left(t, s, z^{*}, w^{*}\right) \\
& \quad+\int_{s}^{t} \alpha_{L 1}\left(s^{\prime}-s\right) O_{L 2}\left(t, s^{\prime}, z^{*}, w^{*}\right) d s^{\prime} \\
& \quad-\int_{0}^{s} \alpha_{L 2}\left(s-s^{\prime}\right) O_{L 2}\left(t, s, z^{*}, w^{*}\right) d s^{\prime}-z_{L}^{*}(s), \\
& \frac{\partial}{\partial s} O_{R 2}\left(t, s, z^{*}, w^{*}\right)=i \omega_{2} O_{R 2}\left(t, s, z^{*}, w^{*}\right) \\
& \quad+i \Omega_{0} O_{L 2}\left(t, s, z^{*}, w^{*}\right) \\
& \quad+\int_{s}^{t} \alpha_{R 1}\left(s^{\prime}-s\right) O_{R 2}\left(t, s^{\prime}, z^{*}, w^{*}\right) d s^{\prime} \\
& \quad-\int_{0}^{s} \alpha_{R 2}\left(s-s^{\prime}\right) O_{R 2}\left(t, s, z^{*}, w^{*}\right) d s^{\prime}-z_{R}^{*}(s),
\end{aligned}
$$

with final conditions at $s=t: O_{L 1}\left(t, t, z^{*}, w^{*}\right)=$ $c_{1}, O_{R 1}\left(t, t, z^{*}, w^{*}\right)=c_{2}, O_{L 2}\left(t, t, z^{*}, w^{*}\right)=c_{1}^{\dagger}$, and $O_{R 2}\left(t, t, z^{*}, w^{*}\right)=c_{2}^{\dagger}$.

Define $\mathcal{Q}_{\lambda n}(t, s) \equiv \mathcal{M}\left\{O_{\lambda n}\left(t, s, z^{*}, w^{*}\right) P_{t}\right\}$, where $\lambda=$ $L, R$, and $n=1,2$. From Eqs. (B1) and (B2), the evolution equations for the operator functions $\mathcal{Q}_{\lambda n}(t, s)$ can be derived as

$$
\begin{aligned}
\frac{\partial}{\partial s} \mathcal{Q}_{L 1}(t, s)= & -i \omega_{1} \mathcal{Q}_{L 1}(t, s)-i \Omega_{0} \mathcal{Q}_{R 1}(t, s) \\
& -\int_{0}^{s} \alpha_{L 1}\left(s-s^{\prime}\right) \mathcal{Q}_{L 1}\left(t, s^{\prime}\right) d s^{\prime} \\
& +\int_{s}^{t} \alpha_{L 2}\left(s^{\prime}-s\right) \mathcal{Q}_{L 1}\left(t, s^{\prime}\right) d s^{\prime} \\
& +\int_{0}^{t} \alpha_{L 2}^{*}\left(s-s^{\prime}\right) \mathcal{Q}_{L 2}^{\dagger}\left(t, s^{\prime}\right) d s^{\prime} \\
\frac{\partial}{\partial s} \mathcal{Q}_{R 1}(t, s)= & -i \omega_{2} \mathcal{Q}_{R 1}(t, s)-i \Omega_{0} \mathcal{Q}_{L 1}(t, s) \\
& -\int_{0}^{s} \alpha_{R 1}\left(s-s^{\prime}\right) \mathcal{Q}_{R 1}\left(t, s^{\prime}\right) d s^{\prime} \\
& +\int_{s}^{t} \alpha_{R 2}\left(s^{\prime}-s\right) \mathcal{Q}_{R 1}\left(t, s^{\prime}\right) d s^{\prime} \\
& +\int_{0}^{t} \alpha_{R 2}^{*}\left(s-s^{\prime}\right) \mathcal{Q}_{R 2}^{\dagger}\left(t, s^{\prime}\right) d s^{\prime}
\end{aligned}
$$


and

$$
\begin{aligned}
\frac{\partial}{\partial s} \mathcal{Q}_{L 2}(t, s)= & i \omega_{1} \mathcal{Q}_{L 2}(t, s)+i \Omega_{0} \mathcal{Q}_{R 2}(t, s) \\
& +\int_{s}^{t} \alpha_{L 1}\left(s^{\prime}-s\right) \mathcal{Q}_{L 2}\left(t, s^{\prime}\right) d s^{\prime} \\
& -\int_{0}^{s} \alpha_{L 2}\left(s-s^{\prime}\right) \mathcal{Q}_{L 2}\left(t, s^{\prime}\right) d s^{\prime} \\
& +\int_{0}^{t} \alpha_{L 1}^{*}\left(s-s^{\prime}\right) \mathcal{Q}_{L 1}^{\dagger}\left(t, s^{\prime}\right) d s^{\prime}, \\
\frac{\partial}{\partial s} \mathcal{Q}_{R 2}(t, s)= & i \omega_{2} \mathcal{Q}_{R 2}(t, s)+i \Omega_{0} \mathcal{Q}_{L 2}(t, s) \\
& +\int_{s}^{t} \alpha_{R 1}\left(s^{\prime}-s\right) \mathcal{Q}_{R 2}\left(t, s^{\prime}\right) d s^{\prime} \\
& -\int_{0}^{s} \alpha_{R 2}\left(s-s^{\prime}\right) \mathcal{Q}_{R 2}\left(t, s^{\prime}\right) d s^{\prime} \\
& +\int_{0}^{t} \alpha_{R 1}^{*}\left(s-s^{\prime}\right) \mathcal{Q}_{R 1}^{\dagger}\left(t, s^{\prime}\right) d s^{\prime} .
\end{aligned}
$$

Correspondingly, the final conditions of $\mathcal{Q}_{\lambda n}(t, s)$ at $s=t$ are given by $\mathcal{Q}_{L 1}(t, t)=c_{1} \rho_{t}, \mathcal{Q}_{L 2}(t, t)=c_{1}^{\dagger} \rho_{t}$, $\mathcal{Q}_{R 1}(t, t)=c_{2} \rho_{t}$, and $\mathcal{Q}_{R 2}(t, t)=c_{2}^{\dagger} \rho_{t}$.

According to Eqs. (B3) and (B4) and the final conditions of the operator functions $\mathcal{Q}_{\lambda n}(t, s), \mathcal{Q}_{\lambda n}(t, s)$ should take the forms

$$
\begin{aligned}
\mathcal{Q}_{\lambda 1}(t, s)= & A_{\lambda 1}^{*}(t, s) c_{1} \rho_{t}+A_{\lambda 2}^{*}(t, s) \rho_{t} c_{1}+A_{\lambda 3}^{*}(t, s) c_{2} \rho_{t} \\
& +A_{\lambda 4}^{*}(t, s) \rho_{t} c_{2}, \\
\mathcal{Q}_{\lambda 2}(t, s)= & B_{\lambda 1}(t, s) \rho_{t} c_{1}^{\dagger}+B_{\lambda 2}(t, s) c_{1}^{\dagger} \rho_{t}+B_{\lambda 3}(t, s) \rho_{t} c_{2}^{\dagger} \\
& +B_{\lambda 4}(t, s) c_{2}^{\dagger} \rho_{t},
\end{aligned}
$$

where $\lambda=L, R$, and the final conditions of $A_{\lambda j}(t, s)$ and $B_{\lambda j}(t, s)$ are given as follows: $A_{L 1}(t, t)=A_{R 3}(t, t)=$ $B_{L 2}(t, t)=B_{R 4}(t, t)=1$, and $A_{\lambda j}(t, t)=0, B_{\lambda j}(t, t)=0$ for other $\lambda$ and $j$. Here $A_{\lambda j}(t, s)$ and $B_{\lambda j}(t, s)$ satisfy the following integro-differential equations:

$$
\begin{aligned}
& \frac{\partial}{\partial s} A_{L j}(t, s)-i \omega_{1} A_{L j}(t, s)-i \Omega_{0} A_{R j}(t, s) \\
& \quad+\int_{0}^{s} \beta_{L}\left(s-s^{\prime}\right) A_{L j}\left(t, s^{\prime}\right) d s^{\prime}=U_{L j}(t, s), \\
& \frac{\partial}{\partial s} A_{R j}(t, s)-i \omega_{2} A_{R j}(t, s)-i \Omega_{0} A_{L j}(t, s) \\
& \quad+\int_{0}^{s} \beta_{R}\left(s-s^{\prime}\right) A_{R j}\left(t, s^{\prime}\right) d s^{\prime}=U_{R j}(t, s),
\end{aligned}
$$

and

$$
\begin{gathered}
\frac{\partial}{\partial s} B_{L j}(t, s)-i \omega_{1} B_{L j}(t, s)-i \Omega_{0} B_{R j}(t, s) \\
\quad+\int_{0}^{s} \beta_{L}\left(s^{\prime}-s\right) B_{L j}\left(t, s^{\prime}\right) d s^{\prime}=V_{L j}(t, s), \\
\frac{\partial}{\partial s} B_{R j}(t, s)-i \omega_{2} B_{R j}(t, s)-i \Omega_{0} B_{L j}(t, s) \\
\quad+\int_{0}^{s} \beta_{R}\left(s^{\prime}-s\right) B_{R j}\left(t, s^{\prime}\right) d s^{\prime}=V_{R j}(t, s),
\end{gathered}
$$

with

$$
\begin{aligned}
& U_{L j}(t, s)=\int_{0}^{t} \alpha_{L 2}\left(s-s^{\prime}\right) h_{L 1}\left(t, s^{\prime}\right) d s^{\prime}, \\
& U_{R j}(t, s)=\int_{0}^{t} \alpha_{R 2}\left(s-s^{\prime}\right) h_{R 2}\left(t, s^{\prime}\right) d s^{\prime}, \quad \text { for } j=1,2, \\
& U_{L j}(t, s)=\int_{0}^{t} \alpha_{L 2}\left(s-s^{\prime}\right) h_{L 2}\left(t, s^{\prime}\right) d s^{\prime}, \\
& U_{R j}(t, s)=\int_{0}^{t} \alpha_{R 2}\left(s-s^{\prime}\right) h_{R 1}\left(t, s^{\prime}\right) d s^{\prime}, \quad \text { for } j=3,4,
\end{aligned}
$$

and

$$
\begin{aligned}
& V_{L j}(t, s)=\int_{0}^{t} \alpha_{L 1}\left(s-s^{\prime}\right) h_{L 1}\left(t, s^{\prime}\right) d s^{\prime}, \\
& V_{R j}(t, s)=\int_{0}^{t} \alpha_{L 1}\left(s-s^{\prime}\right) h_{R 2}\left(t, s^{\prime}\right) d s^{\prime}, \quad \text { for } j=1,2, \\
& V_{L j}(t, s)=\int_{0}^{t} \alpha_{L 1}\left(s-s^{\prime}\right) h_{L 2}\left(t, s^{\prime}\right) d s^{\prime}, \\
& V_{R j}(t, s)=\int_{0}^{t} \alpha_{R 1}\left(s-s^{\prime}\right) h_{R 1}\left(t, s^{\prime}\right) d s^{\prime}, \quad \text { for } j=3,4 .
\end{aligned}
$$

In Eqs. (B8) and (B9), $h_{L n}(t, s)$ and $h_{R n}(t, s)$, with $n=$ 1,2 , are solutions of the two coupled integro-differential equations:

$$
\begin{aligned}
\frac{\partial}{\partial s} h_{L n}(t, s)= & i \omega_{1} h_{L n}(t, s)+i \Omega_{0} h_{R n}(t, s) \\
& +\int_{s}^{t} \beta_{L}\left(s-s^{\prime}\right) h_{L n}\left(t, s^{\prime}\right) d s^{\prime} \\
\frac{\partial}{\partial s} h_{R n}(t, s)= & i \omega_{2} h_{R n}(t, s)+i \Omega_{0} h_{L n}(t, s) \\
& +\int_{s}^{t} \beta_{R}\left(s-s^{\prime}\right) h_{R n}\left(t, s^{\prime}\right) d s^{\prime}
\end{aligned}
$$

with final conditions at $s=t: h_{L 1}(t, t)=h_{R 1}(t, t)=1$, and $h_{L 2}(t, t)=h_{R 2}(t, t)=0$. Here $\beta_{\lambda}\left(s-s^{\prime}\right)$ is defined by

$$
\beta_{\lambda}\left(s-s^{\prime}\right) \equiv \alpha_{\lambda 1}\left(s^{\prime}-s\right)+\alpha_{\lambda 2}\left(s-s^{\prime}\right) .
$$

Using the obtained $\mathcal{Q}_{\lambda n}(t, s)$ in Eqs. (B5), one can calculate

$$
\begin{aligned}
& \mathcal{M}\left\{\bar{O}_{\lambda n}\left(t, z^{*}, w^{*}\right) P_{t}\right\}=\int_{0}^{t} d s \alpha_{\lambda n}(t-s) \mathcal{Q}_{\lambda n}(t, s), \\
& \mathcal{M}\left\{P_{t} \bar{O}_{\lambda n}^{\dagger}(t,-z,-w)\right\}=\int_{0}^{t} d s \alpha_{\lambda n}(t-s) \mathcal{Q}_{\lambda n}^{\dagger}(t, s) .
\end{aligned}
$$

Substituting Eq. (B12) into Eq. (23), we then obtain the exact master equation in Eq. (38) for the doublequantum-dot system. 
[1] J. Q. You and F. Nori, Nature (London) 474, 589 (2011), and references therein.

[2] B. L. Hu, J. P. Paz, and Y. Zhang, Phys. Rev. D 45, 2843 (1992).

[3] R. P. Feynman and F. L. Vernon, Ann. Phys. (N.Y.) 24, 118 (1963).

[4] L. Diósi, N. Gisin, and W. T. Strunz, Phys. Rev. A 58, 1699 (1998).

[5] W. T. Strunz, L. Diósi, and N. Gisin, Phys. Rev. Lett. 82, 1801 (1999).

[6] T. Yu, L. Diósi, N. Gisin, and W. T. Strunz, Phys. Rev. A 60, 91 (1999).

[7] W. T. Strunz and T. Yu, Phys. Rev. A 69, 052115 (2004).

[8] J. Jing and T. Yu, Phys. Rev. Lett. 105, 240403 (2010).

[9] C. J. Broadbent, J. Jing, T. Yu, and J. H. Eberly, Ann. Phys. (N.Y.) 327, 1962 (2012).

[10] J. Jing, X. Zhao, J. Q. You, and T. Yu, Phys. Rev. A 85, 042106 (2012).

[11] P. Hedegard and A. O. Caldeira, Phys. Scripta 35, 609 (1987); Y. C. Chen, J. Stat. Phys. 47, 17 (1987).

[12] M. W. Y. Tu and W. M. Zhang, Phys. Rev. B 78, 235311 (2008).

[13] J. S. Jin, M. W. Y. Tu, W. M. Zhang, and Y. J. Yan, New J. Phys. 12083013 (2010).

[14] S. A. Gurvitz and Ya. S. Prager, Phys. Rev. B 53, 15932
(1996).

[15] T. H. Stoof and Yu. V. Nazarov, Phys. Rev. B 53, 1050 (1996).

[16] G. Kießlich, E. Schöll, T. Brandes, F. Hohls, and R. J. Haug, Phys. Rev. Lett. 99, 20602 (2007).

[17] A. Cottet, W. Belzig, and C. Bruder, Phys. Rev. B 70, 115315 (2004).

[18] H. S. Goan, G. J. Milburn, H. M. Wiseman, and H. B. Sun, Phys. Rev. B 63, 125326 (2001).

[19] H. Haug and A. P. Jauho, Quantum Kinetics in Transport and Optics of Semiconductors (Springer, Berlin, 1998).

[20] J. Dalibard, Y. Castin, and K. Mølmer, Phys. Rev. Lett. 68, 580 (1992).

[21] J. Piilo, S. Maniscalco, K. Härkönen, and K.-A. Suominen, Phys. Rev. Lett. 100, 180402 (2008).

[22] T. Yu, Phys. Rev. A 69, 062107 (2004).

[23] A. Atland and B. Simons, Condensed Matter Field Theory, 2nd ed. (Cambridge University Press, Cambridge, 2010).

[24] J. Q. You and H. Z. Zheng, Phys. Rev. B 60, 13314 (1999).

[25] W. F. Shi, X. Zhao and T. Yu, arXiv: 1203.2219. 